\title{
Path Selection for Quantum Repeater Networks
}

\author{
Rodney Van Meter • Takahiko Satoh • Thaddeus D. Ladd • \\ William J. Munro • Kae Nemoto
}

Received: date / Accepted: date

\begin{abstract}
Quantum networks will support long-distance quantum key distribution (QKD) and distributed quantum computation, and are an active area of both experimental and theoretical research. Here, we present an analysis of topologically complex networks of quantum repeaters composed of heterogeneous links. Quantum networks have fundamental behavioral differences from classical networks; the delicacy of quantum states makes a practical path selection algorithm imperative, but classical notions of resource utilization are not directly applicable, rendering known path selection mechanisms inadequate. To adapt Dijkstra's algorithm for quantum repeater networks that generate entangled Bell pairs, we quantify the key differences and define a link cost metric, seconds per Bell pair of a particular fidelity, where a single Bell pair is the resource consumed to perform one quantum teleportation. Simulations that include both the physical interactions and the extensive classical messaging confirm that Dijkstra's algorithm works well in a quantum context. Simulating about three hundred heterogeneous paths, comparing our path cost and the total work along the path gives a coefficient of determination of 0.88 or better.
\end{abstract}

Keywords Quantum communication, Quantum repeater, Dijkstra, path selection.

R. Van Meter

Keio University, Fujisawa, Japan E-mail: rdv@sfc.wide.ad.jp

T. Satoh

University of Tokyo, Japan E-mail: satoh@is.s.u-tokyo.ac.jp

T. D. Ladd

Stanford University, Palo Alto, CA, and National Institute of Informatics, Tokyo, Japan

W. J. Munro

NTT Basic Research Labs, Atsugi, Japan

K. Nemoto

National Institute of Informatics, Tokyo, Japan

\section{Introduction}

A routing algorithm chooses a path on a graph, and consists of two parts: a definition for the cost of a single link, and a function for calculating the cost of a path based on those link costs, allowing us to extend a single point-to-point channel to a richer network. Dijkstra's Shortest Path First algorithm, for example, takes a simple scalar cost for each link and treats the sum of link costs as a cost for a candidate path [1]. The emerging field of quantum communication has, to date, experimentally demonstrated the basic principles of entangled quantum networking [2, 3, 4, 5, 6], and laid the theoretical foundations of creating long-distance, high-quality entanglement [7,8,9], but topologically has considered primarily channels and linear networks, leaving us with an urgent need for a path selection mechanism as quantum networks develop.

Quantum key distribution (QKD) is probably the most prominent use of quantum communication, and commercial products are available [10,11,12]. In QKD, quantum effects (and a large dose of classical statistics) are used to detect the presence of an eavesdropper on the channel. QKD generates streams of shared, secret, random bits, which can then be used to key a cryptographic session, such as an IPsec tunnel [13,14, 15, 16]. To extend QKD over distances exceeding a certain limit (a few hundred $\mathrm{km}$ in telecom fiber today) or to create networks without direct links between all pairs of nodes, we can use trusted relay nodes and direct optical switching. Such metropolitan-area QKD networks have already been demonstrated in Boston, Vienna, Geneva, Tokyo, Hefei, and other cities [11, 13, 16, 17].

Long-distance QKD without the trusted relay nodes, as well as most other distributed quantum applications, can be achieved through the use of entanglement [18, 19] (entanglement and other italicized vocabulary will be explained in more depth in Sec. 2). Physical links can create entan- 
glement over short distances; in multi-hop networks, many short-distance entangled states are used to forge the end-toend entangled states consumed by applications. Complex, irregular network topologies with hops of varying length and quality, from quantum LANs through MANs to WANs, will result as quantum systems grow. The devices that will bridge long distances and route connections through networks are called quantum repeaters [8].

Entangled states have uses beyond QKD. The quantum communications toolkit includes such building blocks as quantum Byzantine agreement, distributed leader election, secure multi-party communication, and distributed arithmetic for building large-scale quantum applications [20,21, 22, 23].

Thus, heterogeneous quantum networks hover in our future, but substantial theoretical and engineering hurdles remain to be cleared. The first, theoretical studies on entangled quantum networks focused primarily on an abstract model consisting of a linear chain of repeaters, with a power of two number of hops of identical length and quality [8, 24]. Recent work [25, 26, 27] has targeted more realistic chains of repeaters, relaxing those constraints. Here, we analyze the behavior of more complex network topologies, as in Fig. 1. In a network of heterogeneous links and irregular topology, path selection affects both the performance of individual connections and global network load. Purify-and-swap quantum repeaters are a theoretically well-developed approach, and are strong candidates for field deployment, and we use them as our subject.

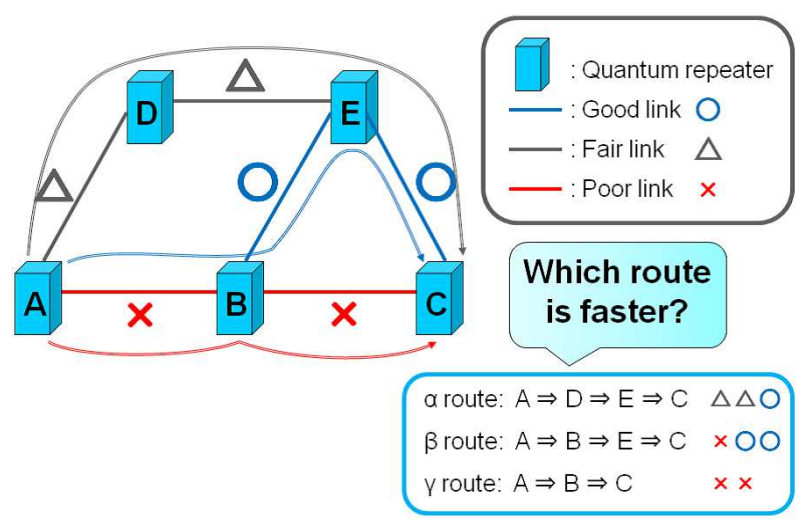

Fig. 1 Path selection is a critical problem in all networks; in quantum networks, due to the delicacy of quantum information, it may determine whether or not a connection can be made successfully.

In this paper, we apply Dijkstra's algorithm for ranking candidate paths to quantum repeater networks [1]. We evaluate our algorithm for its ability to select a path that both maximizes the throughput of end-to-end connections and minimizes global work for this class of repeater. Detailed simulations of both the physical interactions and the clas- sical messaging confirm reasonable agreement between the calculated path cost and the expected throughput.

Our proposed link cost is the inverse of the throughput of the link, measured in Bell pairs per second of a particular fidelity. Other candidates for link cost, including lowerlevel metrics such as the number of laser pulses and quantum measurement operations, are found to be useful for evaluating the total work actually consumed on a path, but are poor metrics for prioritizing a link because they reflect the physical link characteristics but not the system factors that are equally important influences on end-to-end performance.

We present the results of two sets of simulations of various paths using four different qualities of links. The first set of forty-six paths vary in length from one to nine hops, while the second set covers 256 link combinations in fourhop paths. Across both data sets, the coefficient of determination is 0.88 or better between the path cost and the total work performed (counted as the number of quantum measurements performed along the whole path), supporting our choice of link cost and the effectiveness of Dijkstra for this type of quantum network. Comparing the results of pairs of simulations, the path with the lower cost also has higher throughput in more than $80 \%$ of all tested cases. We demonstrate that, in direct analogy to classical networks, the performance of a quantum path will be limited by the throughput of the bottleneck link, while total work is a function of both the path length and the quality of all the links.

To build a complete argument, we discuss the differences between quantum and classical networks and the difficulties encountered (Sec. 2). After defining the problems in path selection and proposing several solutions (Sec. 3), we evaluate those solutions via simulations that answer a series of specific questions about the behavior of quantum repeater networks (Sec.4).

\section{Difficulties: Differences Between Quantum and Classical Networks}

So far in this paper, we have discussed quantum communication technologies and outlined where we apply classical techniques to the quantum problems. However, we have not specifically articulated the fundamental differences that make the merger of classical and quantum networking concepts less than straightforward. There are both theoretical results and practical reasons for believing that the answers to our questions require thought, rather than simply asserting that classical and quantum networks can use the same solutions.

The differences stem from several sources: the engineering difficulties of creating and protecting quantum states and the real-time decay of quantum information; the impact of (probabilistic) photon loss; and the fundamentally probabilistic nature of some quantum operations. These issues 
manifest themselves both locally and globally, requiring in some cases additional classical messages to be exchanged, further exacerbating the problems.

Perhaps most importantly, we cannot make independent copies of quantum information, a fact known as the no cloning theorem [28]. In classical systems, data is copied as it is transmitted; if the data is lost, retransmission is done with no harm beyond a performance penalty. With only a single copy of quantum data, we are forced to treat that data with extra care. Generally, quantum networks build generic states which are then used to teleport precious data [7]. To understand teleportation, we must first discuss entanglement; after teleportation and its uses, we can complete the role of quantum repeater by discussing imperfect quantum states.

\subsection{Entangled States}

Quantum communication involves the sending and receiving of quantum information, encoded in either the state of an individual photon, or a quantum state of a stronger optical or microwave pulse. The state may be sent through a waveguide such as an optical fiber, or through free space, even via satellite [5, 12, 16, 29,30,31]. A transmitted state may stand alone, or be part of a larger, entangled quantum state.

Entanglement, which Einstein referred to as "spooky action at a distance", is the condition of a quantum state in which the states of subsystems are not independent: operations on one part of the system can affect the state of other parts, regardless of physical distance. This effect does not violate the principle of relativity, because it cannot be used to transmit information instantaneously; interpreting the meaning of the distant parts requires the use of classical information acquired during the local quantum operations.

That classical information about the quantum states is acquired via measurement of the states. Measuring a single quantum bit (qubit) produces one classical bit whose value depends on the details of the quantum state, and destroys any entanglement of the qubit with others. The actual measurement mechanism is of course technology-dependent, but often involves interferometric optical setups with very sensitive photodetectors, allowing polarization or other characteristics of an optical state to be determined, detecting a single photon or measuring the direction of spin of a single electron. For the quantum states of interest here, the classical information gained is guaranteed by quantum mechanics to be random, and must be transmitted to the remote site, which of course can only be done at the speed of light.

The particular type of entanglement we require is called a Bell pair. Although entanglement is location independent, when we refer to a Bell pair in this paper, we mean a geographically distributed Bell pair consisting of one qubit at one location entangled with a qubit at another location. Entanglement comes in many forms and can involve more than two parties, but in this paper we focus on the two-party Bell pairs both as intermediate resources and the end goal of the communication session delivered to the application layer.

Quantum communication systems use entanglement in various ways, and for different purposes. Some uses require that the end nodes store quantum data in memory for extended periods of time (many round-trip times); others utilize quantum states of light, but measure the state directly upon receipt without storing, converting the quantum state to a classical value. One of the greatest challenges in quantum communication is dealing with memory lifetimes that are low compared to round-trip latencies.

\subsection{Teleportation and Entanglement Swapping}

To teleport a qubit, we begin with a Bell pair with one end held at our source and the other held at the destination. Local entangling operations are performed on the data qubit and the source-end Bell pair qubit, then both are measured, giving two classical bits of information. Those two classical bits are transmitted to the destination, which then performs local quantum operations on its Bell-pair qubit, dependent on the values of those bits. This recreates the original data qubit at the destination.

Entanglement swapping (Fig.2) splices two short-distance Bell pairs into one longer-distance Bell pair: in Fig.2 $(A \leftrightarrow$ $B)+(B \leftrightarrow C) \Rightarrow(A \leftrightarrow C)$ combines the two left gold arcs to form the green one above. Swapping is a form of teleportation; it can be viewed as using the $(B \leftrightarrow C)$ Bell pair to teleport the $B$ end of the $(A \leftrightarrow B)$ pair to $C$. Classical information must be sent to both ends of the new entangled connection. Managed carefully, each swapping step can double the span of our entangled Bell pair.

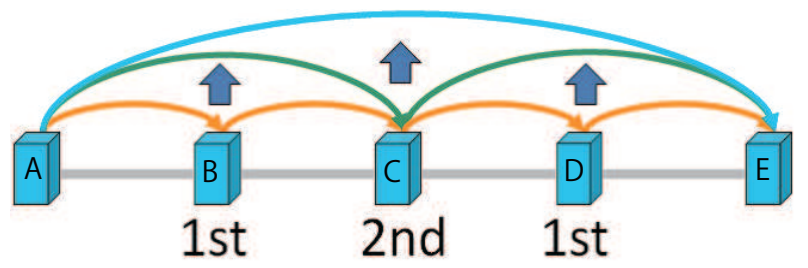

Fig. 2 Symmetric nested entanglement swapping, splicing shorterdistance entangled pairs to create longer-distance entanglement, is accomplished easily in $n$ steps for $2^{n}$ hops of the same quality. The legend and arrows show the order in which entanglement swapping is performed. The arcs represent entanglement over different distances, created via the swapping of shorter entangled pairs.

\subsection{Imperfect Quantum States}

In real systems, Bell pairs are imperfect, and have a characteristic known as fidelity, which represents the accuracy 
of our knowledge about the quantum state, with $F=1.0$ representing perfection. Mathematically, the fidelity is the amount of overlap between the state we intended to create and an average over an ensemble of states we actually created, either experimentally or in simulation, and is defined as $F=\left\langle\Psi^{+}|\rho| \Psi^{+}\right\rangle$, where $\rho$ is the density matrix representation of the state we have, and $\left|\Psi^{+}\right\rangle$is the Bell state we are trying to create. In experiments, fidelity is determined statistically using large numbers of repetitions, creating a quantum state and measuring it to see if the state was properly made and stored. In simulations, various imperfections are numerically introduced on the state creation, processing, or storage to mimic the experimental procedure. By simulating smaller or larger imperfections, we can guide the experimental focus toward the most critical problems, and establish fidelity targets which experimentalists must meet for systems to be operationally viable. In network operations, the results of a set of experiments run during link initialization will be used to track expected behavior of the system.

Purification takes two imperfect $(F<1.0)$ Bell pairs that span one or more hops and attempts to create one higherfidelity Bell pair with fidelity $F^{\prime},\left(F<F^{\prime}<1.0\right)$. The biggest disadvantage of purification is that it is probabilistic, requiring two-way classical communication, tying up buffer memory resources and reducing throughput.

\subsection{Quantum Repeaters}

The devices that create Bell pairs over a distance are called quantum repeaters, building on the concept of teleportation. Because teleportation is imperfect, some form of error control is necessary. In this paper, we focus on the form of repeater we will call the purify-and-swap approach. Purifyand-swap repeaters, as the name suggests, perform two main functions: entanglement swapping to lengthen connections and purification to manage errors [8,24]. Bell pairs are consumed during the course of teleportation, purification and entanglement swapping; thus, the primary job of a repeater is to continually produce new ones. From Bell pairs spanning shorter distances, we can create end-to-end Bell pairs, which are then given to applications. "Throughput" can be defined as Bell pairs per second of a given fidelity, for a given path.

We have defined a protocol stack for purify-and-swap repeaters [32], consisting of three layers: Entanglement Control (EC) at the link level, and Purification Control (PC) and Entanglement Swapping Control (ESC). The latter two operate at arbitrary distance. They can be composed recursively [33] to build high-fidelity Bell pairs in a nested fashion across any nodes in a network, but doing so requests resources which depend critically on the route chosen in the network. The operations of these protocol stack elements are therefore the ones we vary in the present work.
As we shall see in Sec. 4.2, the low probability of entanglement success requires that link-layer operations be acknowledged. Coupled with very limited buffering resources, this results in behavior that differs from most classical networks. Quantum memory is very expensive, simply because of the (usually cryogenic) hardware needed to maintain quantum states for timescales comparable to photon time-of-flight, and to connect that hardware to photons. The fidelity of data in quantum memory in most technologies decays very quickly, adding a hard real-time component to communication, or a demand for strong error correction. This decay may make congestion control a different, even more severe, problem than it is in classical networks.

For a classical path consisting of a series of identical links, throughput (at least in the ideal, sustained case) can match the throughput of a link, independent of the number of hops. However, the maximum throughput of a symmetric chain of purify-and-swap repeaters declines polynomially with length, due to the need for additional purification [24]. Thus, it may be desirable to assign a path cost that grows more than additively.

When operated to deliver Bell pairs above a specified fidelity threshold (typically related to the fidelity threshold for executing quantum error correction), simulations show a stair-step decline in throughput versus hop count [32]. The total work performed grows linearly as the throughput holds steady, then increases suddenly when a "stair" is crossed (see Figs. 3 and 4 in Sec. 4). Likewise, dealing with nonpower-of-two numbers of hops will affect throughput and total work performed in hard-to-predict fashion, due to changes in the swapping and purification patterns.

These principles have been applied within designs for system-area networks, as well as wide-area networks. Interconnects are critical components of quantum computing systems [34, 35, 36, 37]; even some laboratory-sized experiments, much like classical multicomputers, will have multiple levels of interconnect, with different qualities and speeds of connection [38]. These and other large-scale systems will use purified Bell states for data movement. Purification forms a large chunk of the work in these systems as well as in larger networks, and its demands are a commonly-used metric for evaluating systems.

Recent results suggest that using quantum error correction instead of purification opens up new operational approaches more akin to the hop-by-hop behavior of packetswitched networks, and may prove to be more efficient in many cases [25, 26, 27]. These new approaches reduce the demands on memory lifetime and limit the number of return messages required. However, the memory resources required are substantial, far exceeding what will be practical in the near future. The earliest deployments of repeater networks, and perhaps the first commercial products, will likely use the purify-and-swap approach. Thus, we reserve analy- 
sis of our algorithm for other types of networks (and heterogeneous combinations of network types) for future work.

Although some authors have begun speculating about a "quantum Internet", the issues of complex, entangled networks, and heterogeneity at any level above the physical exchange of qubits from one medium to another, are largely open problems [3,9].

\section{Problems and Solutions}

Our goal for this paper is to develop and analyze a routing algorithm for heterogeneous quantum repeater networks, as shown in Fig. 1, Our metric for success, therefore, is agreement between the prospective functioning of a network (represented by detailed simulations) and an easy-to-calculate algorithmic cost: does our algorithm allow us to make effective choices?

As noted above, to develop a routing algorithm, we need a definition for the cost to use a link, a function to calculate a path cost based on a set of link costs, and a goal for the algorithm (e.g., a metric for deciding if the algorithm meets our needs). More precisely, we set as our problems:

PS.1 Choose a goal for the routing algorithm;

PS.2 for a quantum link, identify the characteristics of interest for routing, and reduce them to one number or a small set of numbers that represent the link cost; and

PS.3 for a path (an ordered set of links, with associated costs), define a function that gives a path cost.

To solve these problems, we evaluate the following potential solutions:

PS.1: Goal As the goal for the routing algorithm itself, we choose minimizing work along the path, with attention to the secondary goal of selecting the highest-throughput single path between the defined communication endpoints, measured in Bell pairs per second of the target fidelity. We propose a goal for the system of delivering Bell pairs useful for teleportation, with a target fidelity of $F=0.98$. The phenomena presented here are independent of the exact value chosen, but this value will be adequate for various uses, and allows fairly direct comparison to our prior work [32].

Assessing the work for a particular path is not a simple problem. Intuitively, we want our measure of work to reflect use of some scarce resource. We evaluate two candidates discussed as key functions in repeaters above: total measurement operations and total pulse count.

Without some reasonable idea of global traffic (which we don't yet have) 11, evaluation of global success is difficult,

\footnotetext{
1 However, for heterogeneous traffic matrices, a gravity model might be a reasonable first guess [39], as the human patterns driving quantum communication are expected to be similar to those for classical communication.
}

so as a first step we are evaluating the correlation between total work on the path, achieved connection throughput and our definition of path cost.

PS.2: Link Cost By analogy with the "transceiver time" definition often used with OSPF, we are exploring several link cost definitions. Simply assessing the clock speed at which laser pulses can be emitted is clearly an inadequate link cost metric in a system where the fidelity of the output state is important and the probability of success depends on the characteristics of the link. Prior to execution of our experiments, differing intuitions led one author to suggest Bell pair generation time and another to suggest the number of measurements; eventually we had a list of five candidates:

Loss the loss in the channel, in decibels;

InvTrans the inverse of the transmittance of the channel ( $1 / T$, where the transmittance $T$ is the percentage of photons received through the path);

Pulse the number of laser pulses used to create an entangled Bell pair of a high fidelity over a single hop, corresponding to the number of uses of the transmitter (each fixed-time);

Meas the number of measurement operations used to create an entangled Bell pair of a high fidelity over a single hop (this differs from the above because (a) some pulses are discarded rather than accepted when receiving qubit resources are busy, and (b) measurements are used in entanglement swapping and purification); and

BellGenT the inverse of the throughput of the single link, when run as a single-hop system, measured in seconds per Bell pair.

This list can be divided into two groups: the first two candidates are simply physical characteristics of the link that can be measured easily, while the latter three require simulation or monitoring of the link to determine. The first two differ by a logarithmic factor; InvTrans corresponds to addition while Loss in $\mathrm{dB}$ corresponds to multiplication of cost when placed in a Dijkstra context. While at first glance it may appear desirable to have such an easily-determined link cost, by tying the cost so directly to the physical mechanism, the definition may not transfer well when links of heterogeneous physical technologies are involved.

Pulse and Meas seem to correspond most closely to the "transceiver time" definition, but BellGenT incorporates system factors and may give more accurate estimates for little additional complexity. (Although the technical details are very different, these could be considered roughly analogous to the raw transceiver rate, the throughput of a flow-controlled link, and the throughput of a reliable link-layer protocol, in terms of the increasing functionality present.)

PS.3: Path Cost Function The principle hypothesis of our paper is that Dijkstra's algorithm can be used as-is, with 
an appropriate choice of link cost. More formally,

$C_{\text {path }}=\sum_{i} c_{i}, i \in\{P\}$,

where $\{P\}$ is the set of links in a path and $c_{i}$ is the cost for link $i$. When it is necessary to distinguish among the link cost candidates, we will refer to them as Dijkstra/BellGenT and similarly.

\section{Simulation and Results}

Our goal is to determine the range of conditions under which Dijkstra correctly selects the lowest-work path and highestthroughput path, and when it selects some reasonable approximation. We also wish to articulate the conditions under which lowest work and highest throughput are not the same path and when the algorithm does not pick the lowest-work path. We wish to examine whether we consider those to be acceptable cases, or if the algorithm is "failing". This can be achieved by creating a set of candidate paths and comparing the ordering established by Dijkstra/BellGenT and the ordering according to simulation of the whole path.

In this section, we begin by asking a set of questions about the behavior of heterogeneous paths 4.1. Next, we describe our simulator, proposed hardware configuration and single-hop simulation results, which both set the Dijkstra parameters and allow us to evaluate our link cost candidates (4.2). After enumerating a set of interesting path candidates (4.3), we can answer our questions (4.4), and use those answers to solve the research problems posed in Sec. 3 4.5.

\subsection{The Behavior Questions}

We use the simulator to assess the behavior of systems that are too complex to solve analytically and cannot yet be built and measured directly. Such simulation results will help to guide the development of actual hardware. We can pose a series of specific questions that will help us understand how heterogeneous paths will behave:

1. How does the number of hops in a path affect throughput and total work? As a specific case, do the throughputs of $2^{n}-1,2^{n}$, and $2^{n}+1$-hop paths vary?

2. How does the number of weak links matter? Does the introduction of a single weak link become a bottleneck, as in classical networks? Does adding a second or third weak link further reduce throughput?

3. How does the position of weak links in the chain affect throughput? Does a weak link at the beginning, in the middle, or at the end differ?

4. Under what circumstances will the path cost mis-order candidate paths with respect to throughput or total work?
For classical systems, we know the answers: although cost increases, (1) the length of the path (in hops) has no effect on the (theoretical) throughput, and (2) the (theoretical) throughput of a path is capped by the throughput of the bottleneck link(s). Question (3) is answered in the negative: ordering does not (or should not) matter, and ordering of path segments should always agree with ordering of the full paths. Question (4) is open-ended, but there are known cases where a lower-performance path can be selected in the service of a larger global goal. The answers to these questions are presented in $\mathrm{Sec} .4 .5$

\subsection{Simulated Hardware and Link Costs}

All of the simulations presented in this paper model the qubus physical entanglement mechanism [40,41]. Qubus uses a strong light pulse to entangle two distant, static qubit memories through a fiber or other waveguide. Alternative approaches use single photons [42] or a small number of photons [43]. In the qubus system, a strong laser pulse first interacts with a physical qubit at the transmitter, such as an atom or a quantum dot held in a cavity, resulting in a small, nonlinear shift in the state of the laser pulse, depending on the state of the qubit. The pulse is sent through a waveguide to the receiver, where it undergoes a similar interaction with another qubit. The pulse is then measured using a technique known as homodyne measurement, giving a classical result. About $40 \%$ of the time, we get a result that tells us only the parity of the two qubits and leaves them entangled.

We use the same simulator as in our prior work, with extensions to support the heterogeneous paths [32,40,43]. The simulator was developed for modeling the quantum-level behavior of a cavity QED system for qubus and two other physical layer candidates, and uses the purification mechanism of Dehaene et al. [44]. The necessary classical messaging is carefully modeled. The simulator consists of about 11,000 commented lines of $\mathrm{C}++$, and the production runs of the simulations presented here consumed about one hundred hours of CPU time on 2.2GHz AMD Opteron CPUs. The simulator is based on well-understood physical equations and is experimentally validated at the lowest levels; as experience with larger-scale quantum networks develops, we plan to continue tracking the agreement of theory, simulation, and experiment. We believe the prospective agreement between a real-world network and our simulations will be more than adequate for the purposes of this paper, but the detailed arguments are largely about physics, and are beyond the scope of this paper.

Figure 3 shows the results of simulating a single qubus hop, with parameters as in Table 1, additional details of the hardware configuration are the same as in Refs. [32, 40,43]. The original qubus mechanism is very sensitive to loss, but 
works well in low-loss situations. This form of qubus repeater fails to work for losses greater than about $5.5 \mathrm{~dB}$ from transmitter qubit to receiver qubit, limiting hop length to about $30 \mathrm{~km}$ over high-quality optical fiber at telecom wavelengths. Other types of physical link, including variants of the qubus mechanism [43], will work over longer distances and with higher fidelity. By choosing to simulate basic qubus links, we can see very clearly the impact of purification and low-fidelity entanglement. As future work, we plan to confirm the behavior of Dijkstra with other physical link types.

For the multi-hop simulations, we choose four specific points as example links, as shown in Table 2. The four link types chosen are marked in the figure with the corresponding symbols. The terms "standard", "good", "fair", and "poor" are relative to this simulation only, not indicative of all possible physical quantum link types. As can be seen from this table and Figure 3, even small differences in loss have a large and uneven impact on throughput, suggesting that not only is the utility of the proposed link costs Loss and InvTrans rather technology-specific, but even in the isolated case of qubus they may be poor metrics.

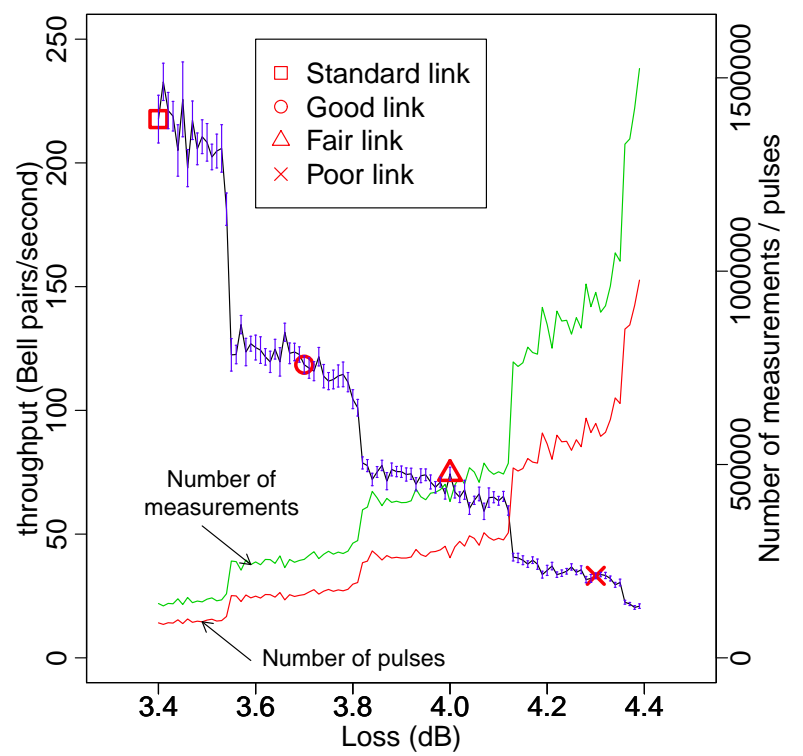

Fig. 3 Single-hop simulated throughput versus loss using the parameters in Table 1 used to define linkcost. The four link types chosen for simulation of more complex paths are marked on the black/blue curve with the corresponding symbols. The stair-step behavior is due to the increasing number of purification rounds as the initial fidelity declines due to increasing loss. The details of this curve are very specific to the qubus technology we simulate, but the principles are general.

Although Bell pairs are symmetric and can be used to teleport data in either direction, at the physical level, links are naturally dependent on a transmitter and a receiver. Receivers dynamically select a qubit to attempt to entangle,
Table 1 Link cost simulation parameters

\begin{tabular}{|c|c|}
\hline \multicolumn{2}{|c|}{ Qubus Quantum Repeater } \\
\hline $\begin{array}{c}\text { Number of qubits } \\
\text { per repeater link connection }\end{array}$ & $\begin{array}{c}25 \text { transmitter, } \\
25 \text { receiver }\end{array}$ \\
\hline $\begin{array}{c}\text { Number of qubits } \\
\text { teleported (length of simulation) } \\
\text { Final target fidelity }\end{array}$ & 200 qubits \\
\hline \multicolumn{2}{|c|}{ Optical Fiber } \\
\hline \multicolumn{2}{|c|}{ Length } \\
\hline Signal loss & $3.4 \sim 4.4 \mathrm{~dB} / 20 \mathrm{~km}$ \\
\hline
\end{tabular}

based on availability at the time of pulse reception, much as any network interface chooses a buffer in which to place an incoming packet. The inherent round-trip latency in receiving acknowledgement of entanglement success or failure (the Entanglement Control (EC) protocol layer) means that the qubits at the transmitter must spend long periods of time in which they are nominally marked as busy, but no useful forward progress can be be made. Thus, links with the same physical loss but different numbers of qubits at the transmitter and receiver will behave differently, as shown in Table 3 .

Although the throughput varies by a factor of two for different configurations, the number of pulses and number of measurements to teleport 200 qubits does not vary significantly except for the clearly misconfigured 100/25 case. For the conditions simulated, about 450 pulses and 700 measurements (including basic entanglement, swapping and purification) are required to teleport one qubit. Although these two measures are arguably more direct representations of cost, this important throughput difference affects our notion of a preferred path.

Reasoning about the behavior of only a single hop and confirming the results via simulation allow us easily to eliminate two of our prospective link cost candidates (Loss and InvTrans). Two others (Pulse and Meas) remain good measures of total work, but the results shown in Tab. 3 cast doubt on their viability as candidates for link cost when the goal is to achieve high throughput. Thus, we settle on BellGenT as our link cost metric.

\subsection{Simulated Path Candidates}

The candidate paths we choose to examine are designed to answer the questions in Sec. 4.1. We simulated forty-six paths of one to nine hops in various patterns, as well as all $4^{4}=256$ four-hop combinations for the four chosen link types. The forty-six paths are enumerated along the bottom of Fig. 4. We have simulated many more paths with a variety of link conditions over the course of this experiment, notably very long, homogeneous paths (up to 2,048 hops). The findings from other simulations do not contradict the 
Table 2 The link configurations and five candidates for link cost. The cost candidates to the left of the first double line are physical characteristics of the channel, while the others are the results of single-hop simulations. Simulation parameters are as in Table 1

\begin{tabular}{|c|c|c|c|c|c|c|c|c|c|c|}
\hline Link & Loss & InvTrans & Pulse & per-t'port & (norm) & Meas & per-t'port & (norm) & T'put & BellGenT \\
\hline Standard ( $\square)$ & $3.4 \mathrm{~dB}$ & 2.19 & 90441 & 452 & 1 & 140519 & 702 & 1 & 217.7 & 1 \\
\hline Good $(\bigcirc)$ & $3.7 \mathrm{~dB}$ & 2.34 & 163628 & 818 & 1.80 & 254691 & 1237 & 1.76 & 118.4 & 1.83 \\
\hline Fair $(\triangle)$ & $4.0 \mathrm{~dB}$ & 2.51 & 258852 & 1294 & 2.86 & 404117 & 2020 & 2.87 & 74.3 & 2.93 \\
\hline Poor $(x)$ & $4.3 \mathrm{~dB}$ & 2.69 & 606278 & 3031 & 6.70 & 945247 & 4276 & 6.72 & 33.1 & 6.57 \\
\hline
\end{tabular}

Table 3 Throughput depends on the number of available qubits at both transmitter and receiver. Due to the round-trip latency in the Entanglement Control (EC) protocol for acknowledging successful entanglement, having more qubits at the transmitter boosts throughput. Simulations are for a single hop with parameters as in Table 1 Throughput and confidence interval (std. dev.) are determined by a linear fit to teleportation completion times.

\begin{tabular}{|c|c|c|c|c|}
\hline Xmtr & Rcvr & Throughput & Pulses & Meas \\
\hline 25 & 25 & $237 \pm 8$ & 80587 & 125905 \\
25 & 50 & $213 \pm 8$ & 92071 & 143582 \\
50 & 25 & $436 \pm 14$ & 99708 & 142723 \\
50 & 50 & $456 \pm 14$ & 89533 & 139506 \\
100 & 25 & $462 \pm 22$ & 199240 & 138543 \\
100 & 50 & $956 \pm 31$ & 92221 & 137828 \\
100 & 100 & $984 \pm 32$ & 88694 & 138145 \\
\hline
\end{tabular}

results presented in this paper, which were chosen to clearly show the effects of interest.

\subsection{Answering Our Behavior Questions}

Figures 4, 5] and 6 show our simulation results for the paths. In Fig. 4, the throughput for each specific path can be seen, as well as the two measures of total work, Pulse and Meas. In Fig. 5. throughput is plotted against the Dijkstra-calculated path cost using BellGenT as our cost metric, and in Fig. 6 the total work measures are plotted against calculated path cost. Figures 7 and 8 plot the results for all 256 four-hop paths we simulated.

Perhaps the most important factor to note about the behavior we see is the discrete nature of changes to throughput, due to the discrete nature of purification and entanglement swapping and our choice to establish a particular threshold for final acceptance of an end-to-end Bell pair. In many circumstances, minor changes force an additional round of purification, generally causing a 50\% reduction in throughput.

As the number of hops (Q.1) increases, our results show a stair-step phenomenon in throughput, but not necessarily at powers of two: the decline of fidelity resulting in additional rounds of purification does not move in concert with entanglement swapping. For Standard $\square$ paths, we see steps at the 2 nd and 7 th hops.

Examining the symbols in Fig. 5 shows clearly the existence of a bottleneck link phenomenon (Q. 2): with one low-quality link in the path, the quality of the other links is

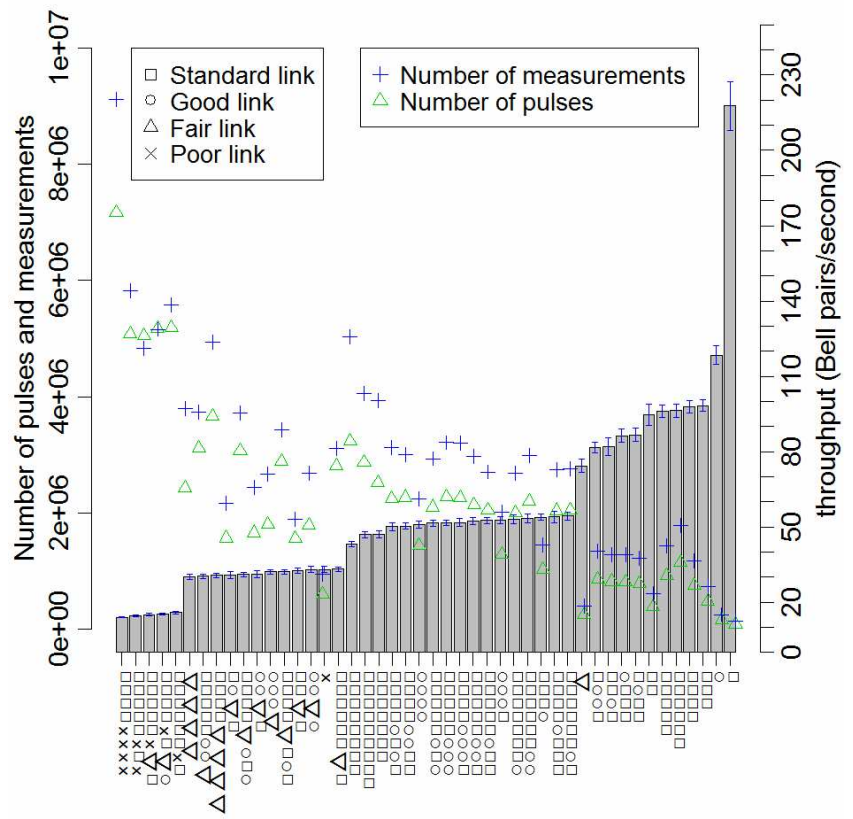

Fig. 4 Total number of pulses $(\triangle)$ and measurements $(+)$ for fortysix of our candidate paths (right scale). The paths vary in length from one to nine hops. They are ordered left to right according to ascending throughput, plotted using bars (left scale). The legend below the graph shows the individual path configurations; $\square, \bigcirc, \triangle$ and $\times$ represent our standard, good, fair, and poor links, respectively. The stair-step behavior reflects increasing numbers of rounds of purification.

almost irrelevant, as can be seen by the clustering of each type of data point (e.g., $\triangle$ at about 30 Bell pairs/second in Fig. 5 and the concentration of $\times$ links at the left edge of Fig. 4). The various paths can largely, though not entirely, be grouped according to the throughput of the slowest link in the path. The most eye-catching anomalies in Fig. 5 are the single $\bigcirc, \triangle$ and $\times$ marks above and to the right of their respective clusters. These are the single-hop paths of the corresponding link type, indicating that we do not have a pure bottleneck phenomenon. The bottleneck plus the polynomial decline in performance as the number of hops grows work in combination determine to the path performance. In most cases for the longer paths, adding a second link of the same quality as the bottleneck link does not result in a statistically significant reduction in throughput, but optimization of the path becomes more difficult. In a few cases, our optimization fails to find an acceptable pattern, and an additional round of 


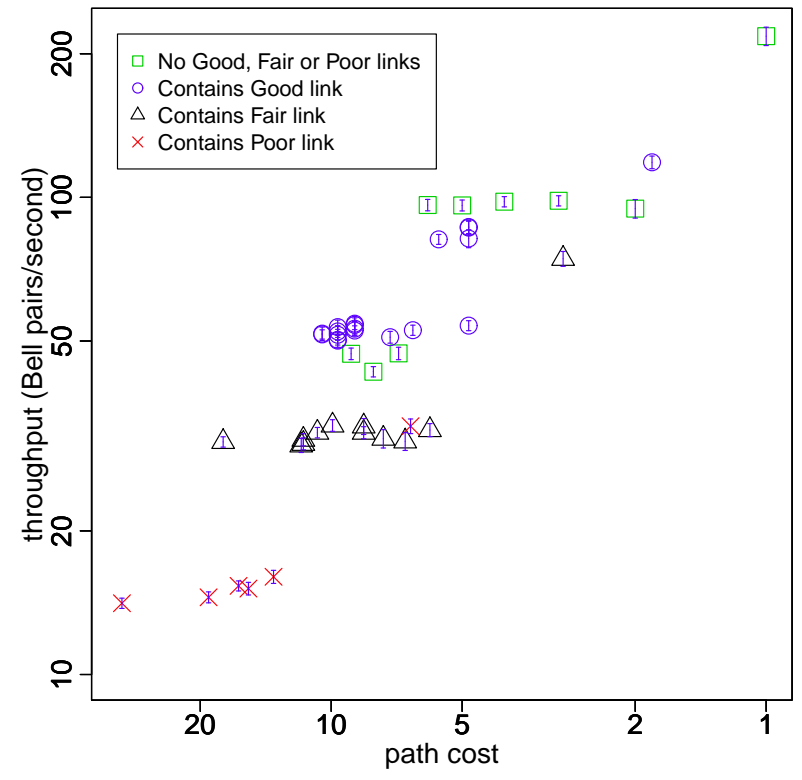

Fig. 5 Throughput versus BellGenT path cost for forty-six of our candidate paths. Each path is represented by the symbol for the weakest type of link in the path. The clustering of each type of data point shows clearly that throughput is limited by the bottleneck link. The length of the vertical bar (mostly contained within each symbol) shows the std. dev. of the throughput.

purification becomes necessary. In particular, four-hop paths with $\bigcirc$ as the bottleneck link(s) are split into two plateaus at around 80 Bell pairs/second and 50 Bell pairs/second. However, the total work shows strong correlation even for these cases.

Despite this general bottleneck behavior, the weak link position (Q.3) is a more subtle one. Among our simulations, we found a single case where the bottleneck position produced different results. With one Good link and three Standard ones, having the Good link at the left end of the path resulted in 53 Bell pairs/second, whereas the other three paths produced 82 to 87 Bell pairs/second. The low-throughput case required an extra round of purification before one entanglement swapping operation. As the path hovers near a threshold demanding an extra round of purification, optimization of the path usage becomes more difficult; three paths successfully did so, while the fourth didn't. Thus, we must answer that the weak link position may have an impact on throughput, depending on our ability to effectively use the path.

Dijkstra/BellGenT occasionally mis-orders (Q. 4 ) pairs of path candidates with respect to throughput. Comparing the 256 four-hop paths we simulated, there are 32,640 possible pairs, of which 1,230 had the same path cost. Of the pairs with different costs, $82.6 \%$ of the time Dijkstra/BellGenT chooses the higher-throughput path of the pair (the "correct" choice), and $17.4 \%$ of the time it chooses the lower-

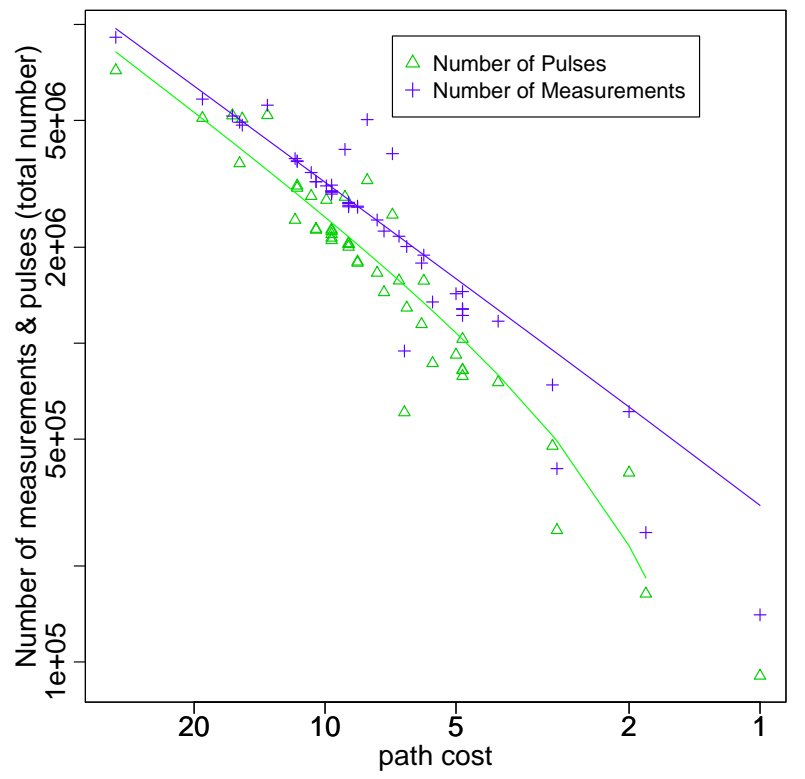

Fig. 6 Total work, measured in Pulses $(\triangle)$ and Measurements $(+)$, versus BellGenT path cost for forty-six of our candidate paths. The coefficient of determination of each linear fit is 0.88 , showing that our path cost is a strong predictor of total work.

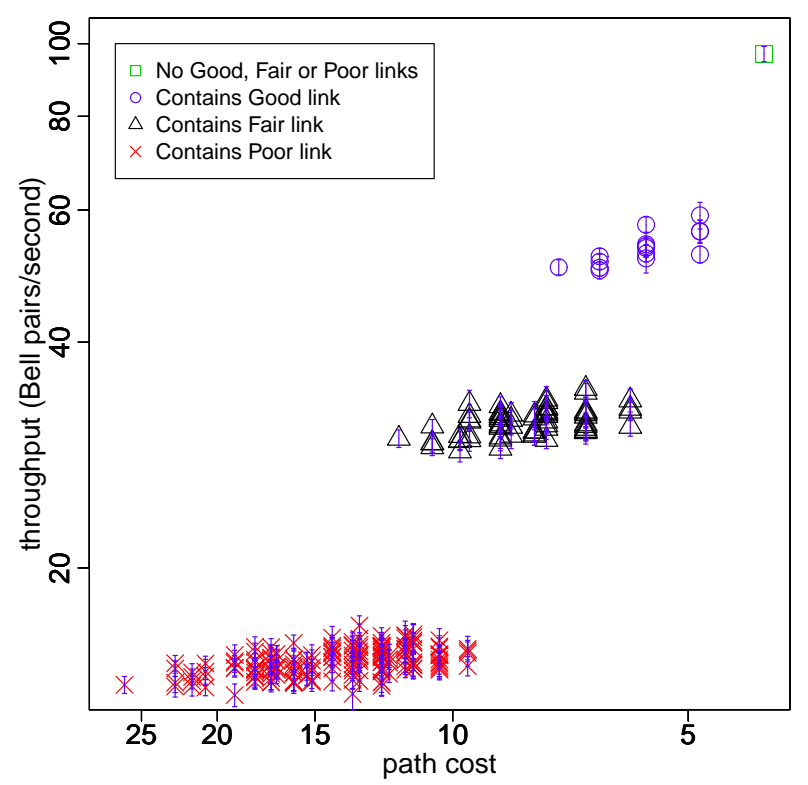

Fig. 7 Throughput versus BellGenT path cost for all 256 four-hop candidate paths. Each path is represented by the symbol for the weakest type of link in the path. The clustering of each type of data point shows clearly that throughput is limited by the bottleneck link.

throughput path (the "incorrect" choice). In only $5 \%$ of those mis-ordering cases was the difference in throughput more than $10 \%$. For the 46 variable-length paths, the rate of "correct" choices was similar, $81.6 \%$, but the impact of those choices is higher, with a throughput penalty of $25 \%$ or more in almost half of the "incorrect" cases. 


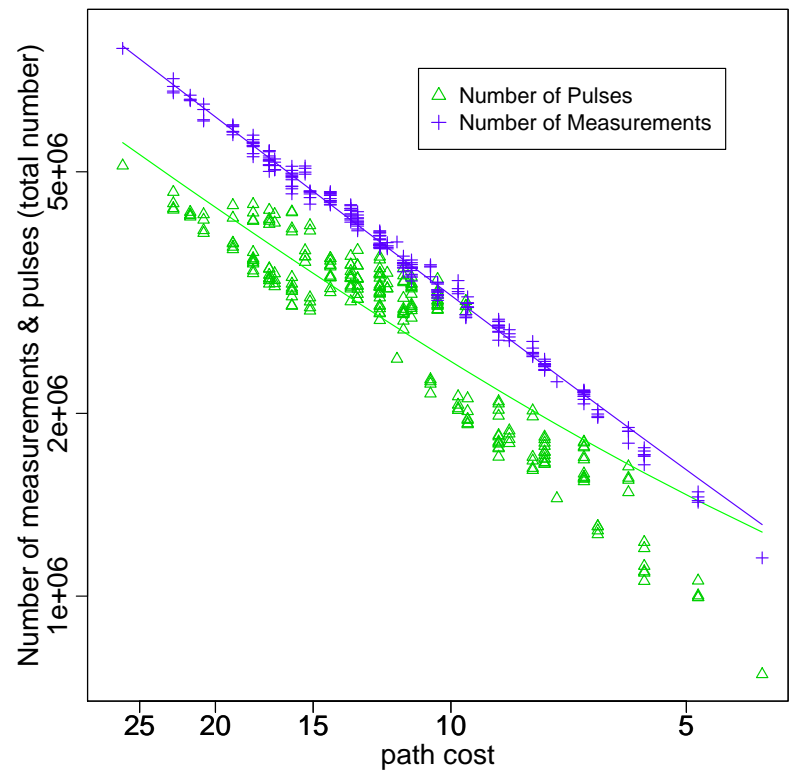

Fig. 8 Total work, measured in pulses $(\triangle)$ and measurements $(+)$, versus BellGenT path cost for all 256 four-hop candidate paths, with linear fits. The coefficient of determination for the number of pulses is 0.81 , and for the number of measurements is 0.99 .

The most remarkable case of incorrect ordering we found is the pair of paths $\square \times \square \square \square \square \square \square$ (throughput: $16.0 \pm 0.51$ cost: 13.57) and $\triangle \triangle \triangle \triangle \square \square \square \square$ (throughput: $30.7 \pm 0.77$ cost: 15.72). The higher-cost path has twice the throughput of the lower-cost one. Examining Fig. 4 shows that the total work (in pulses or measurements) is quite similar. This isolated case would suggest that the bottleneck Poor link might warrant even a higher link cost than 6.57, however, in all of the other cases we examined, it has worked well.

The performance and work are both dominated by purification costs. The use of a given path must be optimized as a whole; our current solution is brute force, trying a large number of possibilities. In a large majority of cases, optimization of that process for a path is straightforward and robust. However, a noticeable minority of cases require delicate adjustments to the entanglement swapping settings. Further automated optimization of this process may result in both better performance and agreement between path cost and work.

For total work, we find a coefficient of determination of 0.88 for both pulses and measurements for the forty-six variable-length paths. For the 256 four-hop paths, we find a coefficient of 0.81 for pulses and 0.99 for measurements.

We can summarize the behavior as follows: Performance is determined by the number of rounds of purification used anywhere on the path; the number of rounds is dominated by the bottleneck link. Work, however, is spread across the entire path in rough proportion to relative link quality.

\subsection{Solving Our Problems}

With the simulator results and answers to our questions in hand, we are now prepared to assess the solutions we proposed in Sec. 3, and determine whether Dijkstra/BellGenT meets our goal of developing an acceptable routing algorithm for quantum repeater networks.

We have seen that the highest-throughput path is also strongly correlated to measures of work, including total pulse count and number of measurements. In some pathological cases, number of measurements is a better metric, and across a broad range of cases it matches well with our chosen link cost below. Our solution to Path Selection Problem PS.1 is therefore: to minimize total work along the path, using number of measurements as the metric.

We saw in Sec. 4.2 that defining BellGenT as our link cost metric is likely to better suit our purposes than Pulse, Meas, Loss, or InvTrans, allowing us to propose a solution to Problem PS.2: seconds per Bell pair.

Our simulation results presented in the previous subsections indicate clearly that we can usually, if not always, correctly predict the highest-throughput, lowest-work path from among a set of candidates. Dijkstra/BellGenT solves Problem PS.3 well: path cost is the scalar sum of link costs.

Intuitively, we can see why this works well, as confirmed by the simulations: BellGenT is directly related to the number of purification rounds needed, and hence the amount of work on a link. Despite the concerns we expressed earlier about the nonlinear amount of work as the number of hops grows, the predictive ability for ordering paths remains strong. Thus, we can assert that even as the use of complex repeater networks evolves, and various traffic patterns arise, Dijkstra/BellGenT likely will remain an effective choice.

\section{Conclusion}

Real-world deployment of networks of quantum repeaters will inevitably be physically heterogeneous, with complex topologies and high- and low-quality links and many possible paths through the networks, rather than an idealized, homogeneous power-of-two number-of-hops.

In this paper, we have investigated critical problems in the use of purify-and-swap repeater networks. Our focus has been on path selection, and the need for a routing algorithm. An acceptable routing algorithm must be an easyto-calculate metric that reliably chooses a reasonable, if not optimal, path.

Our results show that, despite many important differences, quantum repeater networks behave similarly to classical networks in useful ways, but the classical principles cannot be applied without thought. Via detailed physical simulation of both the physics and the classical messaging proto- 
cols, we have investigated several variants and explored the range of conditions under which these principles apply.

We can predict the throughput of a connection based primarily on the bottleneck link in the path, while the total work, in number of operations performed (pulses or measurements), increases with the addition of other, non-bottleneck links, much as in a classical network path. Applying a form of Dijkstra's algorithm with the inverse throughput of each hop as the link cost (Dijkstra/BellGenT) results in strong correlation between our easily-calculated path cost and actual throughput, and between cost and total work. This is achieved with reasonable computational effort, allowing us to recommend the use of Dijkstra.

In future work, we expect to confirm the general behavior of Dijkstra with other physical-layer repeater types, adding single-photon [45] and low-photon number [43] to the qubus systems explored here. Our simulator is capable of modeling the important factor of finite quantum memory lifetimes [46], but the sheer additional combinatoric complexity that would have come from including variation of this parameter in both the simulations and algorithmic arguments in this paper prevents us from presenting these results.

Perhaps the most important open question is whether these results apply to error-corrected, rather than purified, repeater networks [25,26, 27]. Demonstrating the applicability of Dijkstra to both purify-and-swap and error-corrected repeater networks would be a strong indicator of the universality of our results. We expect that initial network demonstrations will be purify-and-swap, moving toward error-corrected but perhaps with a long period of coexistence.

The results presented here, concerning routing within a network of uniform technology but disparate operating conditions, are part of a larger program attempting to unify various quantum networking approaches within a single framework, covering inter-networking between heterogeneous physical technologies as well as radically different error correction approaches, routing, multiplexing approaches and quality of service, and distributed applications. With these results, we expect to take advantage of the knowledge accumulated in half a century of classical networking research and operation to create a strong quantum network architecture, guide the experimental focus for the development of repeaters, implement real-world quantum protocols and accelerate deployment of quantum networks.

\section{Acknowledgements}

This work was supported by the Japan Society for the Promotion of Science, KAKENHI 21500020. TDL was partially supported by the Ministry of Education, Culture, Sports, Science and Technology-Japan and the National Institute of Information and Communications Technology. WJM and KN acknowledge support from the National Institute of Information and Communications Technology.

\section{References}

1. EW Dijkstra. "A note on two problems in connexion with graphs," Numerische Mathematik, 1(1):269-271, 1959.

2. Chin-Wen Chou, Julien Laurat, Hui Deng, Kyung Soo Choi, Hugues de Riedmatten, Daniel Felinto, and H. Jeff Kimble. "Functional quantum nodes for entanglement distribution over scalable quantum networks," Science, 316(5829):1316-1320, 2007.

3. H. J. Kimble. "The quantum Internet," Nature, 453:1023-1030, June 2008.

4. R. Reichle, D. Leibfried, E. Knill, J. Britton, RB Blakestad, JD Jost, C. Langer, R. Ozeri, S. Seidelin, and DJ Wineland. "Experimental purification of two-atom entanglement," Nature, 443(7113):838-41, 2006.

5. Toshiyuki Tashima, Tsuyoshi Kitano, Şahin Kaya Özdemir, Takashi Yamamoto, Masato Koashi, and Nobuyuki Imoto. "Demonstration of local expansion toward large-scale entangled webs," Phys. Rev. Lett., 105(21):210503, Nov 2010.

6. Z. Zhao, T. Yang, Y.A. Chen, A.N. Zhang, and J.W. Pan. "Experimental realization of entanglement concentration and a quantum repeater," Physical Review Letters, 90(20):207901, 2003.

7. C. H. Bennett, G. Brassard, C. Crépeau, R. Josza, A. Peres, and W. Wootters. "Teleporting an unknown quantum state via dual classical and EPR channels," Physical Review Letters, 70:18951899, 1993.

8. H.-J. Briegel, W. Dür, J.I. Cirac, and P. Zoller. "Quantum repeaters: the role of imperfect local operations in quantum communication," Physical Review Letters, 81:5932-5935, 1998.

9. S. Lloyd, J.H. Shapiro, F.N.C. Wong, P. Kumar, S.M. Shahriar, and H.P. Yuen. "Infrastructure for the quantum Internet," $A C M$ SIGCOMM Computer Communication Review, 34(5):9-20, 2004.

10. C. H. Bennett and G. Brassard. "Quantum cryptography: Public key distribution and coin tossing," In Proc. IEEE International Conference on Computers, Systems, and Signal Processing, pages 175-179. IEEE, December 1984.

11. D. Dodson, M. Fujiwara, P. Grangier, M. Hayashi, K. Imafuku, K. Kitayama, P. Kumar, C. Kurtsiefer, G. Lenhart, N. Luetkenhaus, et al. "Updating Quantum Cryptography Report ver. 1," Arxiv preprint arXiv:0905.4325, 2009.

12. Hoi-Kwong Lo and Yi Zhao. "Quantum cryptography," In Encyclopedia of Complexity and System Science. Springer, 2008. arXiv:0803.2507v4 [quant-ph].

13. Chip Elliott, David Pearson, and Gregory Troxel. "Quantum cryptography in practice," In Proc. SIGCOMM 2003. ACM, ACM, August 2003.

14. Alan Mink, Sheila Frankel, and Ray Perlner. "Quantum key distribution (QKD) and commodity security protocols: Introduction and integration," International Journal of Network Security \& Its Applications (IJNSA), 1(2), July 2009.

15. Shota Nagayama and Rodney Van Meter. "IKE for IPsec with QKD," Internet Draft, draft-nagayama-ipsecme-ipsec-with-qkd00; October 2009, expired April 22, 2010.

16. M Peev et al. "The SECOQC quantum key distribution network in Vienna," New Journal of Physics, 11(7):075001 (37pp), 2009.

17. Teng-Yun Chen, Jian Wang, Hao Liang, Wei-Yue Liu, Yang Liu, Xiao Jiang, Yuan Wang, Xu Wan, Wei-Qi Cai, Lei Ju, Luo-Kan Chen, Liu-Jun Wang, Yuan Gao, Kai Chen, Cheng-Zhi Peng, Zeng-Bing Chen, and Jian-Wei Pan. "Metropolitan all-pass and inter-city quantum communication network," August 2010. arXiv:1008.1508v2 [quant-ph].

18. R Alléaume, F Roueff, E Diamanti, and N Lütkenhaus. "Topological optimization of quantum key distribution networks," New Journal of Physics, 11(7):075002, 2009.

19. A.K. Ekert. "Quantum cryptography based on Bell's theorem," Physical Review Letters, 67(6):661-663, 1991. 
20. M. Ben-Or and A. Hassidim. "Fast quantum Byzantine agreement," In Proceedings of the thirty-seventh annual ACM symposium on Theory of computing, pages 481-485. ACM, 2005.

21. Harry Buhrman and Hein Röhrig. Mathematical Foundations of Computer Science 2003, chapter "Distributed Quantum Computing," pages 1-20. Springer-Verlag, 2003.

22. Ellie D'Hondt. Distributed quantum computation: A measurement-based approach. $\mathrm{PhD}$ thesis, Vrije Universiteit Brussel, July 2005.

23. Seiichiro Tani, Hirotada Kobayashi, and Keiji Matsumoto. "Exact quantum algorithms for the leader election problem," In Proc. STACS 2005: 22nd Annual Symposium on Theoretical Aspects of Computer Science, volume 3404 of Lecture Notes in Computer Science, pages 581-592. Springer-Verlag, 2005.

24. W. Dür and H.J. Briegel. "Entanglement purification and quantum error correction," Rep. Prog. Phys., 70:1381-1424, 2007.

25. Austin G. Fowler, David S. Wang, Charles D. Hill, Thaddeus D. Ladd, Rodney Van Meter, and Lloyd C. L. Hollenberg. "Surface code quantum communication," Phys. Rev. Lett., 104(18):180503, May 2010.

26. Liang Jiang, J. M. Taylor, Kae Nemoto, W. J. Munro, Rodney Van Meter, and M. D. Lukin. "Quantum repeater with encoding," Phys. Rev. A, 79(3):032325, Mar 2009.

27. WJ Munro, KA Harrison, AM Stephens, SJ Devitt, and K. Nemoto. "From quantum multiplexing to high-performance quantum networking," Nature Photonics, 4:792-796, 2010.

28. W. K. Wootters and W. H. Zurek. "A single quantum cannot be cloned," Nature, 299:802, October 1982.

29. A. Fedrizzi, R. Ursin, T. Herbst, M. Nespoli, R. Prevedel, T. Scheidl, F. Tiefenbacher, T. Jennewein, and A. Zeilinger. "Highfidelity transmission of entanglement over a high-loss free-space channel," Nature Physics, 5(6):389-392, 2009.

30. T. Scheidl, R. Ursin, A. Fedrizzi, S. Ramelow, X.S. Ma, T. Herbst, R. Prevedel, L. Ratschbacher, J. Kofler, T. Jennewein, et al. "Feasibility of $300 \mathrm{~km}$ quantum key distribution with entangled states," New Journal of Physics, 11(085002):085002, 2009.

31. Paolo Villoresi, Thomas Jennewein, Fabrizio Tamburini, Markus Aspelmeyer, Cristian Bonato, Rupert Ursin, Claudio Pernechele, Vincenza Luceri, Giuseppe Bianco, Anton Zeilinger, and Cesare Barbieri. "Experimental verification of the feasibility of a quantum channel between Space and Earth," New Journal of Physics, 10:033038, 2008

32. Rodney Van Meter, Thaddeus D. Ladd, W. J. Munro, and Kae Nemoto. "System design for a long-line quantum repeater," IEEE/ACM Transactions on Networking, 17(3):1002-1013, June 2009.

33. J.D. Touch, Y.S. Wang, and V. Pingali. "A recursive network architecture," Technical report, ISI, October 2006.

34. Dean Copsey, Mark Oskin, Tzvetan Metodiev, Frederic T. Chong, Isaac Chuang, and John Kubiatowicz. "The effect of communication costs in solid-state quantum computing architectures," In Proceedings of the fifteenth annual ACM Symposium on Parallel Algorithms and Architectures, pages 65-74, 2003.

35. Nemanja Isailovic, Yatish Patel, Mark Whitney, and John Kubiatowicz. "Interconnection networks for scalable quantum computers," In Computer Architecture News, Proc. 33rd Annual International Symposium on Computer Architecture. ACM, June 2006.

36. Tzvetan S. Metodi, Darshan D. Thaker, Andrew W. Cross, Isaac L. Chuang, and Frederic T. Chong. "High-level interconnect model for the quantum logic array architecture," J. Emerg. Technol. Comput. Syst., 4(1):1-28, 2008.

37. Mark Oskin, Frederic T. Chong, Isaac L. Chuang, and John Kubiatowicz. "Building quantum wires: The long and short of it," In Computer Architecture News, Proc. 30th Annual International Symposium on Computer Architecture. ACM, June 2003.
38. Rodney Van Meter, Thaddeus D. Ladd, Austin G. Fowler, and Yoshihisa Yamamoto. "Distributed quantum computation architecture using semiconductor nanophotonics," International Journal of Quantum Information, 8:295-323, 2010.

39. A. Medina, N. Taft, K. Salamatian, S. Bhattacharyya, and C. Diot. "Traffic matrix estimation: Existing techniques and new directions," ACM SIGCOMM Computer Communication Review, 32(4): 174, 2002.

40. T. D. Ladd, P. van Loock, K. Nemoto, W. J. Munro, and Y. Yamamoto. "Hybrid quantum repeater based on dispersive CQED interaction between matter qubits and bright coherent light," New Journal of Physics, 8:184, 2006.

41. P. van Loock, T. D. Ladd, K. Sanaka, F. Yamaguchi, Kae Nemoto, W. J. Munro, and Y. Yamamoto. "Hybrid quantum repeater using bright coherent light," Physical Review Letters, 96:240501, 2006.

42. L. Childress, J.M. Taylor, A.S. Sørensen, and M.D. Lukin. "Faulttolerant quantum communication based on solid-state photon emitters," Physical Review Letters, 96(7):70504, 2006.

43. W. J. Munro, R. Van Meter, Sebastien G. R. Louis, and Kae Nemoto. "High-bandwidth hybrid quantum repeater," Phys. Rev. Lett., 101(4):040502, Jul 2008.

44. J. Dehaene, M. Van den Nest, B. De Moor, and F. Verstraete. "Local permutations of products of Bell states and entanglement distillation," Physical Review A, 67(2):22310, 2003.

45. L. Jiang, J. M. Taylor, and M. D. Lukin. "Fast and robust approach to long-distance quantum communication with atomic ensembles," Phys. Rev. A, 76:012301, Jul 2007.

46. L. Hartmann, B. Kraus, H.-J. Briegel, and W. Dür. "On the role of memory errors in quantum repeaters," Physical Review A, 75:032310, 2007. 